\title{
Evidence for a 2:3 resonance in Sco X-1 kHz QPOs
}

\author{
M. A. Abramowicz ${ }^{1,2,3}$, T. Bulik ${ }^{4}$, M. Bursa ${ }^{3,5}$, and W. Kluźniak ${ }^{1,4,6,7}$ \\ 1 Visiting Professor, Institut d'Astrophysique de Paris, 98bis Bld. Arago, 75014 Paris, France \\ ${ }^{2}$ Université Pierre \& Marie Curie (Paris VI), France \\ 3 Department of Astrophysics, Chalmers University, 412-96 Göteborg, Sweden, e-mail: marek@fy.chalmers . se \\ ${ }^{4}$ Copernicus Astronomical Centre, ul. Bartycka 18, 00-716 Warszawa, Poland, e-mail: bulik@camk.edu.pl \\ 5 Astronomical Institute, Charles University Prague, V Holešovičkách 2 CZ-180 00 Praha 8, Czech Republic \\ e-mail: bursa@sirrah.troja.mff.cuni.cz \\ ${ }^{6}$ Institute of Astronomy, Zielona Góra University, Lubuska 2, 65-265 Zielona Góra, Poland, e-mail: wlodek@camk.edu.pl \\ 7 Centre d'Étude Spatiale des Rayonnements, 9 Av. Colonel-Roche, 31028 Toulouse Cedex 4, France
}

Received 28 June 2002 / Accepted 25 April 2003

\begin{abstract}
We find evidence that the two high frequency QPOs in Sco X-1 are, more often than not, approximately in the 2:3 frequency ratio familiar from studies of black hole candidates (e.g., XTE J1550-564, Remillard et al. 2002). This implies that the double $\mathrm{kHz}$ QPO phenomenon in neutron stars has its origin in properties of strong-field gravity and has little to do with the rotation of a stellar surface or any magnetic field structure anchored in the star.
\end{abstract}

Key words. dense matter - general relativity - stars: neutron - stars: Sco X-1 - X-rays: stars

\section{The larger picture}

Recent X-ray observations showed that in some black holes, as in neutron stars, high frequency quasi-periodic oscillations (QPOs) come in pairs. This in itself suggests that the QPO phenomenon in black holes and in neutron stars may have the same origin. We think that the frequency ratios may be even more revealing.

The frequencies of these QPO pairs in the first three black hole sources where they have been observed (Strohmayer 2001a,b; Remillard et al. 2002) are $300 \mathrm{~Hz}$ and $450 \mathrm{~Hz}$ (in GRO J1655-40), $42 \mathrm{~Hz}$ and $70 \mathrm{~Hz}$ (in GRS 1915+105) and $184 \mathrm{~Hz}$ and $276 \mathrm{~Hz}$ (in XTE J1550-564). Clearly, they are in a 2:3 ratio in two of the sources, and in a 3:5 ratio in the third (Abramowicz \& Kluźniak 2001; Kluźniak \& Abramowicz 2001, 2002; Remillard et al. 2002).

In this paper we examine the published frequency data of the well-studied source Sco X-1, a prototype of bright neutronstar candidates, to see whether the two "kHz" QPO frequencies may be in the ratio of two low integers, such as $2: 3$ or 3:5, as they are in black hole systems. If such a similarity were well established between neutron stars and black holes, it would imply that the "twin" high frequency QPOs originate in a mechanism operating independently of the presence of any stellar surface with its co-rotating magnetic fields. If the high frequency QPOs are fundamentally similar in neutron stars and in black holes, they are all likely to be a manifestation of strong-field gravity.

Send offprint requests to: $\mathrm{W}$. Kluźniak, e-mail: wlodek@camk.edu.pl

\section{The inner disc radius in neutron-star LMXBs}

The $\mathrm{kHz}$ QPOs have fairly similar properties in a wide range of systems. In particular, the highest frequencies have similar values in X-ray bursters and the much brighter sources, such as Sco X-1 (see van der Klis 2000, for a review). This argues against any special mechanism related to accidental properties of the system, such as the value of the magnetic field or of the accretion rate (although many models based on such finely tuned properties have been proposed).

If the oscillation arises in the accretion-disk flow, the orbital frequency sets the scale of expected oscillation frequencies (Bath 1973). In fact, the relevant frequency is the meridional epicyclic frequency. It is identical to the Keplerian frequency for Newtonian $1 / r$ gravity, but in general relativity for accretion disks around neutron stars the meridional epicyclic frequency is typically somewhat smaller than the orbital frequency. Observations of a $\mathrm{kHz}$ frequency then strongly disfavor a geometry of accretion in which the maximum orbital frequency is only a few hundred Hertz. This seems to rule out surface magnetic dipole fields in excess of about $10^{8} \mathrm{G}$ (Kluźniak 1998).

In fact, orbital frequencies in the kilohertz range were expected to be observed in the timing data of neutron stars, on the assumption that magnetic fields are so weak as to play no dynamical role, which allows the presumably geometrically thin discs to be terminated close to the central compact object by effects of strong gravity (Kluźniak et al. 1990). This assumption is now supported by the observed frequency values. In general relativity, characteristic frequencies scale inversely with the mass. If one scales the $70 \mathrm{~Hz}$ QPO frequency of the black 




Fig. 1. The cumulative distribution of the frequency ratio of the twin $\mathrm{kHz}$ QPOs in Sco X-1 and three theoretical models. Dot-dashed line: a uniform distribution in the range $(0.3,0.9)$; dashed line: beat frequency model with upper frequency distributed uniformly in the range $(872 \mathrm{~Hz}, 1081 \mathrm{~Hz})$; thin continuous line: the best fit Lorentzian. Of the three models, only the Lorentzian fits the data.

hole candidate GRS $1915+105$ from $\sim 14 M_{\odot}$ (Greiner et al. 2001) to about one solar mass, one obtains $1 \mathrm{kHz}$, a frequency typical of the high frequencies detected in neutron stars.

Detection of $\mathrm{kHz}$ QPOs thus tends to favour the view that the accretion disks in these neutron star systems terminate close to the marginally stable orbit of general relativity (Lipunov \& Postnov 1984; Kluźniak \& Wagoner 1985; Sunyaev \& Shakura 1986; Kluźniak et al. 1990; Kluźniak \& Wilson 1991; Biehle \& Blandford 1993; Zhang et al. 1997; Kaaret et al. 1997; Kluźniak 1998; Zhang et al. 1998).

\section{Distribution of $\mathrm{kHz}$ QPO frequency ratio}

The general belief seems to be that the distribution of frequency ratios is fairly uniform, as it is for the published frequency pairs in the source GX5-1 (Jonker et al. 2002b), where the frequency ratios span nearly half of the range $(0,1)$ allowed a priori, see Fig. 2. But these GX5-1 results may not reflect the true distribution of frequency ratio, as data were added until an acceptable signal to noise ratio was obtained throughout the observed frequency range. The bias towards a uniform distribution can be eliminated if the QPO fequencies are determined separately in each observation interval (of equal duration). Such a procedure has been carried out for Sco X-1 and 39 frequency pairs have been reported (van der Klis et al. 1997).

The first question to ask is whether the Sco X-1 kHz QPO data are compatible with a uniform distribution of frequency ratios. The observed range of ratios in neutron stars is about $(0.3,0.9)$ and in Fig. 1 we plot the corresponding

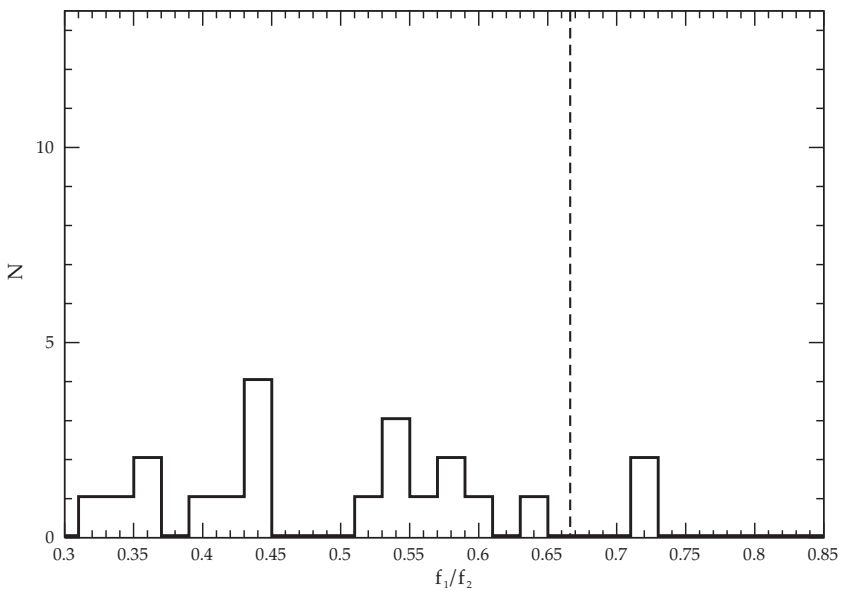

Fig. 2. The frequency ratios of the reported pairs of $\mathrm{kHz}$ QPOs in GX5-1. Also shown is the location of the 2:3 ratio (dashed line). The data is from Jonker et al. (2002b), and may not reflect the relative occurrence rates of frequencies.

"expected" cumulative distribution (dot-dashed line). It is clear that the distribution of $\mathrm{kHz}$ QPO frequency ratios actually observed in Sco X-1 (thick solid line) is completely different.

\section{Is the stellar spin frequency directly observed in the $\mathrm{kHz}$ QPOs of Sco X-1?}

Patterson (1979) pointed out that if there is direct interaction between a structure corotating with the star and a Keplerian accretion disk, a QPO at the beat frequency $\left|1 / P_{*}-1 / P_{\mathrm{K}}\right|$ may result. Here $P_{*}$ is the period of stellar rotation and $P_{\mathrm{K}}$ the period of orbital motion. Patterson was discussing the white dwarf system AE Aqu, and the co-rotating structure was an "illuminating searchlight."

After the discovery of $\sim 40 \mathrm{~Hz}$ QPOs in LMXBs, this idea was adapted to a neutron star rotating at an assumed frequency of $\sim 160 \mathrm{~Hz}$ and beating against a $\sim 200 \mathrm{~Hz}$ Keplerian frequency at the inner edge of an accretion disk presumed to be close to an assumed Alfven radius (Alpar \& Shaham 1985), and given the name "beat-frequency model" (Lamb et al. 1985). But a maximum $200 \mathrm{~Hz}$ Keplerian frequency is incompatible with the more recently discovered $\mathrm{kHz}$ oscillations in the same sources, so the name has been duly changed to "sonic-point model" with one QPO still corresponding to the beat frequency and the other peak to the orbital frequency, both greatly increased (Miller et al. 1998). It has been noted (e.g., Méndez et al. 1998; Jonker et al. 2002a) that, in fact, the difference between the two observed high frequencies in neutron star LMXBs is not constant, as it presumably should be in the beat frequency model. Accordingly, other models were proposed in which the interaction between a Keplerian disk and the stellar magnetosphere gave rise to frequencies in greater concordance with the observations (Osherovich \& Titarchuk 1999).

After the discovery of double high frequency QPOs also in black hole candidates (Strohmayer 2001a,b; Miller et al. 2001; Remillard et al. 2002) the notion that there may be a direct relationship between stellar spin and the presence of two high frequency QPOs has lost its allure. After all, black holes do 
not have a surface and their spin frequency cannot be directly observed. Still, let us examine the expectations of the rotational (beat frequency) model.

If one assumes that the upper frequency, $f_{2}$ varies uniformly (in the range observed in Sco X-1) and the lower fequency is $f_{1}=f_{2}-$ const., the expected cumulative distribution is given by the dashed line of Fig. 1. It does not fit the data.

More generally, when one frequency varies freely over some arbitrary range, and the second differs from the first by a nearly constant value, there would be no reason for the observed frequency ratio to cluster about any particular value, much less a value equal to the ratio of two integers - in any beat frequency model the ratio between the constant stellar rotation rate and the orbital frequency is accidental and cannot be the ratio of two small integers. Even stranger would be a clustering about a value close to that observed in black hole systems, e.g., a 2:3 ratio. And yet, such a clustering is exactly what we find in Sco X-1 (Figs. 1 and 3). We have performed a similar analysis of other neutron star sources, and the peak at about $2 / 3$ ratio is present in those data as well (Fig. 4).

\section{Evidence for the 2:3 frequency ratio in Sco X-1}

We are using the Sco X-1 data of van der Klis et al. (1997), who present the frequencies of the two $\mathrm{kHz}$ QPOs for a number of separate continuous observations of 1 to $3 \mathrm{ks}$ each. The upper frequency, $f_{2}$, varied in the range $(872 \mathrm{~Hz}, 1081 \mathrm{~Hz})$. The difference, $f_{2}-f_{1}$, of the two QPO frequencies was clearly anticorrelated with the frequency $f_{2}$, and varied between about 200 and $300 \mathrm{~Hz}$.

We have computed the ratio $f_{1} / f_{2}$ for each observation of Sco X-1 reported in van der Klis et al. (1997), and find it to be in a much narrower range, $0.643<f_{1} / f_{2}<0.793$, than that of the combined data of all neutron star sources (Figs. 3 and 4). A histogram of the ratios at once makes it evident that the distribution is not uniform, but instead sharply peaked about a central value fairly close to a 2:3 ratio (indicated in Fig. 3 by a vertical dashed line). This conclusion is not affected by the bin width, and is fairly representative of the complete sample.

We are not aware of any observational bias which would favor a selection of those data in which the QPO frequency ratio would cluster about 0.67 , neither are the observers (P. Jonker, private communication).

\section{Statistical significance of the peak in the frequency ratio}

To quantify our claim of clustering about a preferred value of the frequency ratio of " $\mathrm{kHz}$ " QPOs in Sco X-1, we have fit to the observed distribution of frequency ratios a suitably normalized Lorentzian as a trial probability distribution, $\mathrm{d} p / \mathrm{d} r=$ $N\left(r_{0}, \lambda\right) /\left[\left(r-r_{0}\right)^{2}+\lambda^{2}\right]$ where $r_{0}$ and $\lambda$ are free parameters, and $N\left(r_{0}, \lambda\right)$ is the normalization factor. Here, $r \equiv f_{1} / f_{2}$. We use the maximum likelihood method (Bulik et al. 2000) to find the probability distribution that best describes the data, i.e., the most likely value of the two parameters - they are $r_{0}=0.688$ and $\lambda=0.0151$.

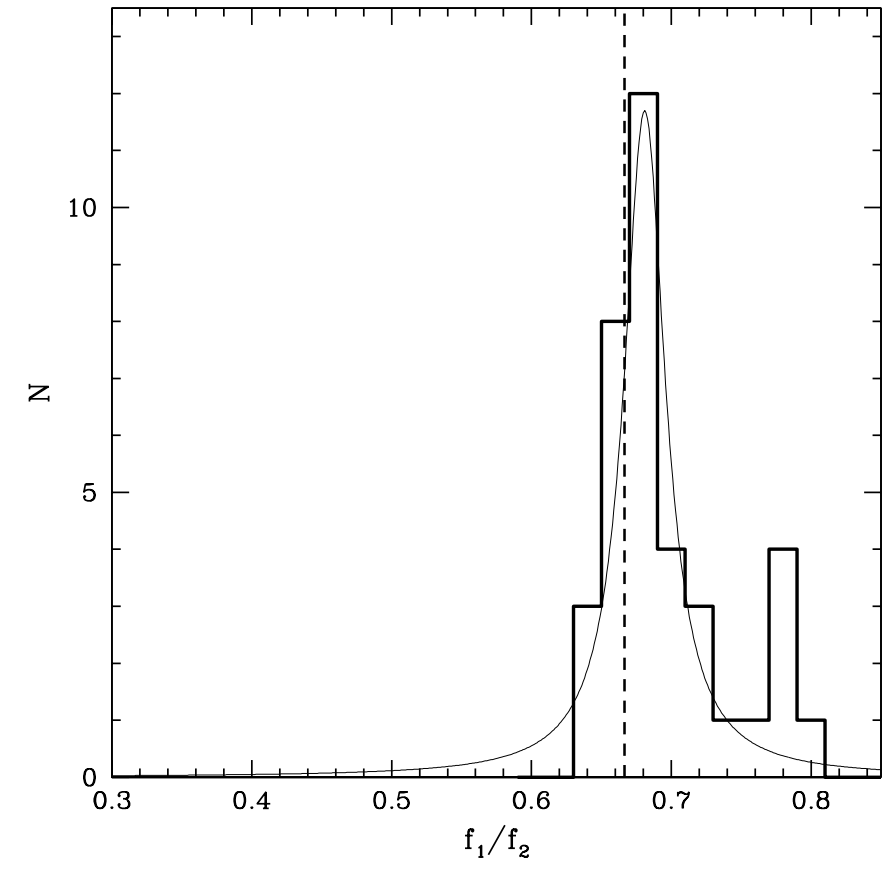

Fig. 3. The observed distribution of frequency ratio of the two $\mathrm{kHz}$ QPOs in Sco X-1. The data is from van der Klis et al. (1997). Also shown is the best fitting Lorentzian.



Fig. 4. The combined distribution of frequency ratios for $4 \mathrm{U} 0614+09$, $4 \mathrm{U}$ 1608-52, 4U 1636-53, 4U 1702-43, 4U 1705-34, 4U 1728-34, $4 \mathrm{U}$ 1735-44, 4U 1820-30, 4U 1915-05, Cyg X-2, GX 17+2, GX 340+0, GX 349+2 (Sco X-2), KS 1731-26, and XTE J2123-058.

The Kolmogorov-Smirnov test confirms that the data could have been drawn from this best-fit Lorentzian distribution, the K-S probability being $p=0.678$. In Fig. 1 we compare the best-fit Lorentzian distribution with the data, and in Fig. 3, we normalize it to the total number of events and superpose it on the histogram.

The K-S probability of a uniform distribution in the range $(0.3,0.9)$ is only $4 \times 10^{-12}$ (dot-dashed line in Fig. 1 ). The beat frequency model described at the end of Sect. 4 (dashed line in Fig. 1), has probability distribution $\mathrm{d} p / \mathrm{d} r \propto 1 /(1-r)^{2}$, and K-S probability of $1.2 \times 10^{-5}$. These two distributions must be rejected. 


\section{A second peak?}

The Sco X-1 data exhibit an additional peak around the value of $r \approx 0.78$. Analysis of Fig. 3d) in van der Klis et al. (1997) suggests that the second peak may be real - the power ratio of the two QPOs departs from the general trend when $f_{1} / f_{2}$ becomes greater than 0.75 (i.e., when $f_{2}>1050 \mathrm{~Hz}$ ). When the QPO frequency ratio is $r=2 / 3$, the power in the upper QPO is about twice that of the lower QPO, then it steadily decreases with increasing frequency ratio until $r \approx 0.75$, when the upper QPO again becomes twice, or thrice, as powerful as the lower one.

Recent observations of the burster 4U 1636-53, support the reality of both peaks in Fig. 3. The frequency difference between the QPOs in 4U 1636-53 seems to undergo an abrupt transition from about $330 \mathrm{~Hz}$ to about $250 \mathrm{~Hz}$ (Jonker et al. 2002a; DiSalvo et al. 2002). On one side of the transition the frequency ratios are $0.662,0.680,0.685,0.725$, i.e., they may well have been sampled from the $2 / 3$ peak in the distribution of Sco X-1; on the other side, the ratios vary between 0.75 and 0.78 .

\section{Interpretation of the results}

Neutron star sources do not typically show a flat distribution of kHz QPO frequency ratios (Figs. 1, 3 and 4). It is hard to escape the conclusion that a resonance is responsible for the distribution of frequency ratios in Sco $X-1$. The $2 / 3$ value, identical to the ratio seen in the black hole candidates GRO J165540 and XTE J1550-564 (whose measured masses are $\sim 6 M_{\odot}$ and $\sim 8 M_{\odot}$ ) is a very strong indication that the high frequency QPOs are a manifestation of strong-field gravity, and not of the presence of a stellar surface with any co-rotating structure.

We note that both the sharp 2:3 frequency ratios in the observed black holes and the broader distribution observed in Sco X-1 with a peak slighty offset from $2 / 3$ seem to be in accord with the theory of epicyclic parametric resonance in relativistic accretion disks (Kluźniak \& Abramowicz 2002), where the higher QPO occurs at the frequency of meridional oscillations, $\Omega_{z}$, in that orbit in which the radial epicyclic frequency is $\Omega_{r}=(2 / 3) \Omega_{z}$. For the Schwarzschild metric this occurs at $r_{2: 3}=5.4 r_{q}=16.2 \mathrm{~km} / M_{\odot}$, and comparison with the observed frequency of $900 \mathrm{~Hz}$ in Sco X-1 would imply that $M=1 M_{\odot}$, with modestly higher values for rotating neutron stars.

Note added 28 April 2003. Recent observations of QPOs in the $2.5 \mathrm{~ms}$ accreting pulsar SAX J1808.4-3658 suggest that the spin of the star may play a role in exciting the resonant modes discussed here (R. Wijnands et al. 2003).

Acknowledgements. We thank Peter Jonker, Michiel van der Klis and Jean-Pierre Lasota for valuable discussion and T. Di Salvo for sharing data prior to publication. This work has been funded in part through the KBN grants 5P03D01721 and 2P03D02117, by CNRS, and by the Swedish Research Council. MAA and WK thank Institut d'Astrophysique de Paris, and WK also CESR, for generous support and hospitality.

\section{References}

Abramowicz, M. A., \& Kluźniak, W. 2001, A\&A, 374, L19

Alpar, M. A., \& Shaham, J. 1985, IAUC, 4046, 2

Bath, G. T. 1973, Nature, Ph.Sc., 246, 84

Biehle, G. T., \& Blandford, R. D. 1993, ApJ, 411, 302

Bulik, T., Kluzniak, W., \& Zhang, W. 2000, A\&A, 361, 153

Di Salvo, T., et al. 2002, submitted

Greiner, J., Cuby, J. G., \& McCaughrean, M. J. 2001, Nature, 414, 522

Jonker, P.G., Méndez, M., \& van der Klis, M. 2002a, MNRAS, 336, L1

Jonker, P. G., van der Klis, M., Homan, J., et al. 2002b, MNRAS, 333, 665

Kaaret, P., Ford, E. C., \& Chen, K. 1997, ApJ, 480, L27

Kluźniak, W. 1998, ApJ, 509, L37

Kluźniak, W., \& Abramowicz, M. A. 2001, Acta Phys. Pol. B, 32, 3605, available at http://th-www.if.uj .edu.pl/acta/

Kluźniak, W., \& Abramowicz, M. A. 2002, A\&A, submitted [astro-ph/0203314]

Kluźniak, W., Michelson, P., \& Wagoner, R. V. 1990, ApJ, 358, 538

Kluźniak, W., \& Wagoner, R. V. 1985, ApJ, 297, 548

Kluźniak, W., \& Wilson, J. R. 1991, ApJ, 372, L87

Lamb, F. K., Shibazaki, N., Alpar, M. A., \& Shaham, J. 1985, Nature, 317,681

Lipunov, V. M., \& Postnov, K. A. 1984, Ap\&SS, 106, 103

Méndez, M., van der Klis, M., \& van Paradijs, J. 1998, ApJ, 506, L117

Miller, M. C., Lamb, F. K., \& Psaltis, D. 1998, ApJ, 508, 791

Miller, J. M., Wijnands, R., Homan, J., et al. 2001, ApJ, 563, 928

Osherovich, V., \& Titarchuk, L. 1999, ApJ, 522, L113

Patterson, J. 1979, ApJ, 234, 978

Remillard, R. A., Muno, M. P., McClintock, J. E. \& Orosz, J. A. 2002, ApJ, 580, 1030

Strohmayer, T. E. 2001a, ApJ, 552, L49

Strohmayer, T. E. 2001b, ApJ, 554, L37

Sunyaev, R. A., \& Shakura, N. I. 1986, SvAL, 12, 117

van der Klis, M. 2000, ARA\&A, 38, 717

van der Klis, M., Wijnands, R. A. D., Horne, K., \& Chen, W. 1997, ApJ, 481, L97

Zhang, W., Strohmayer, T. E., \& Swank, J. H. 1997, ApJ, 482, L167

Zhang, W., Smale, A. P., Strohmayer, T. E., \& Swank, J. H. 1998, ApJ, 500, L171 